EJO 00416

\title{
Fetal Phonocardiography \\ New diagnostic and technical aspects of fetal phonocardiography
}

\author{
Joachim Nagel \\ Department of Biomedical Ehgiheering, University of Miami, \\ Coral Gables, FL, U.S.A.
}

\begin{abstract}
Summary
A new generation of instruments designed to record the fetal phonocardiogram is discussed, as are new methods of time-domain and frequency-domain processing of the fetal phonocardiogram. Using these techniques, detailed information can be obtained about both fetal and maternal cardiovascular conditions.
\end{abstract}

Fetal phonocardiogram; Processing method; Cardiovascular condition

In contrast to adult phonocardiography, not much knowledge exists on the real diagnostic potential of fetal heart sound recording. The main reason for this situation is the poor quality of phonotransducers that have been available in the past, and the consequent severe truncation of the PCG spectrum. Now, with the development of high-quality transducers, the total fetal phonocardiogram can be measured [1,2]. Evaluating its diagnostic potential, however, requires an understanding of the generation of heart sounds, an analysis of what information on the underlying processes is contained in the acoustic signal, and which parts of it can be extracted from the abdominally recorded PCG.

Discussion of this problem can be divided into five different aspects: the recording of the phonocardiogram, the evaluation of cardiac time intervals. the diagnosis of cardiovascular status and of heart diseases, systematic changes of the PCG according to the stage of maturity, and the simultaneous monitoring of the maternal cardiovascular condition from the disturbing signal components. Physical

Correspondence: Dr. J Nagel, Department of Biomedical Engineering. University of Mrami, P.O. Box 248294, Coral Gables, FL 33124, U.S.A. 


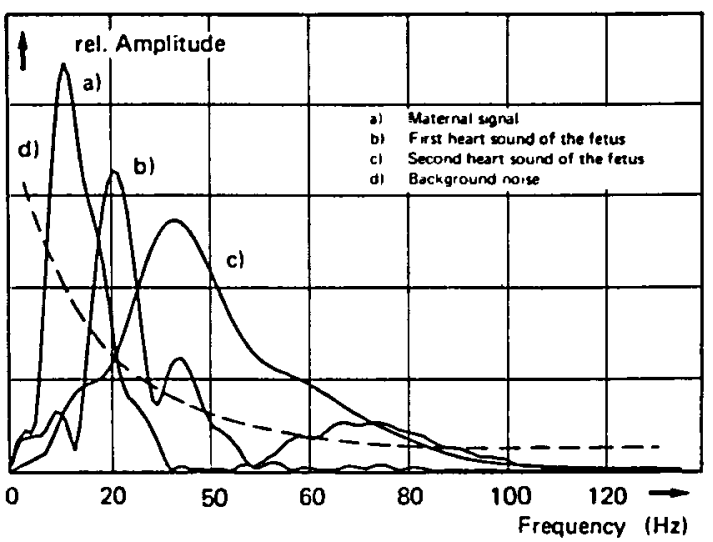

Fig. 1. Spectral power density of the abdominally recorded acoustic signal, separated into its different components: (a) maternal signal, (b) first fetal heart sound, (c) second heart sound, (d) background noise.

and physiological considerations together with clinical experience show that the diagnostic potential of the fetal PCG is much higher than generally supposed, and worth further intensive investigation.

Until now, most studies dealt with the processing of the PCG as a temporal series of distinct events, such as the opening and closing of the heart valves. Consequently the diagnostic potential was restricted to parameters related to the heart rate and the cardiac time intervals. This restriction was exacerbated by the disastrous quality of the available phonotransducers. Conventional narrow-band (typically $80-110 \mathrm{~Hz}$ ) fetal phonocardiography suppresses the low-frequency components of the acoustic signal; however, these contain most of the physiological information (Fig. 1). Of course, signal processing is much easier using conventional transfer characteristics, rather than recording the total acoustic signal $(0.5-250 \mathrm{~Hz})$ which contains many disturbing maternal components. Because of the overlapping spectra, separation of the different components cannot be achieved by simple spectral filtering. More sophisticated techniques are required [3]. Coherent averaging is used to generate templates of the single components. Successive subtraction of these reference signals from the total acoustic signal then results in a complete separation of first and second heart sounds as well as of fetal breathing and maternal sounds. Amplitude variations of the signal, which would lead to non-zero differences, require the templates to be scaled to the actual amplitude of the corresponding components before each subtraction. A running average guarantees the adaptation of the reference signals to variations of the waveforms which might occur during the measuring period. Triggering of the coherent averaging process is achieved by threshold detectors for the maternal component, which is easily detected by its large amplitude, and by spectral tracking, i.e. variable, signal-dependent filtering, which is suitable for identifying first and second heart sounds. A prerequisite for such successful processing of the acoustic signal is a wide-band, low-noise recording of the weak fetal phonocardiogram. 


\section{PCG recording}

Mechanical heart action is accompanied by a displacement and reshaping of the heart, by vibrations of the myocardium, the valves, and the intra-cardiac blood volume, and by eddy flows of blood, all of which contribute to the generation of heart sounds. The traditional technique of PCG recording is the absorption of a part of the mechanical energy that is propagating from the heart to the body surface into a sensor and the conversion of it into an electrical signal. Commonly used transducers are accelerometers, sensing the skin movement, and heavily-weighted stationary microphones, measuring the surface pressure changes. Both transducer types, however, suffer from the same drawback, i.e., the bad coupling to the biological tissue. As the mechanical impedances of tissue and transducer show considerable differences, most of the mechanical energy is reflected back at the boundary surface. Typical values for the transmission coefficient of conventional transducers are $10^{-3}-10^{-2}$. As a result, the signal obtained has a very poor signal-to-noise ratio. This fact and the difficulties of signal processing are the reasons for the necessity of narrow-band filtering of the PCG in previous forms of instrumentation. The resulting loss of essential information may not have been perceived, as electro-acoustic conversion of the filtered PCG produces a sound similar to that of stethoscopic auscultation for the human ear, which, however, is very insensitive at frequencies below $50 \mathrm{~Hz}$. As a consequence of the poor information content of the filtered signals, the diagnostic potential of fetal phonocardiography was limited to the recording of FHR, and even in this application reliability was not always satisfactory.

Substantial improvements in sensor performance can be achieved by matching its mechanical impedance to that of the skin. Two different solutions to this problem have been proposed. Talbert developed a transducer using a piezoelectric cantilever bending bar whose compliance is matched to the mother's abdominal wall. The present author has presented a sensor consisting of a spherical metal plate, also compliance-matched, which transforms, according to the law of Laplace, the pressure acting originally perpendicularly onto its surface into an augmented tangential tension of the plate, which is sensed by a piezoelectric material fixed to the metal plate as a compound element. This phonotransducer shows a slightly better performance at low frequencies. Both sensors, however, are well-suited to record the abdominal acoustic signal over its whole bandwidth with excellent signal-to-noise ratio.

Even better results are obtainable by a non-contacting measurement of the skin's surface motion, which can be realized by either acoustic or optical means. i.e. by ultrasonic or laser Doppler velocimetry, both avoiding the problems of energy transfer and impedance matching. Basic to the Doppler technique is the principle of the frequency shift of waves of any type which are emitted or backscattered from a moving object. This principle is well known from ultrasonics, but, as light is a form of wave energy too, it also shows a Doppler shift of its frequency. Ultrasonic Doppler velocimetry makes use of the well-known piezoelectric crystals as transmitter and receiver. In optical velocimetry, the light source is a laser which guarantees monochromatic and coherent light. To determine the frequency of the 
backscattered light, direct measurements of the frequency shifts cannot be made electronically, as with ultrasonic Doppler, because of the extremely high frequencies of the light. Instead, the Doppler-shifted and the non-shifted light are mixed on the surface of a phototransducer. The mixing of these two components produces optical beating or heterodyning at the Doppler-shifted frequency (or frequency spectrum) and is a direct measure of the skin's motion (i.e., the phonocardiogram). The essential advantages of such contactless PCG recordings are their outstanding sensitivity over the entire frequency band of interest, which is due to the non-loading coupling, the really flat transfer function, and the low liability to interference. Unfortunately the associated instrumentation is rather complex and cumbersome, suitable only for research applications, and not yet for routine clinical use. Future technical developments, however, will certainly solve this problem.

\section{PCG spectral composition}

The heart represents a rather complex oscillatory system, whose spectral power distribution is determined by numerous factors, such as the dimensions of the vibrating tissues (muscle and valves), their properties with respect to elasticity and density, and the physical conditions of vibrational excitation, especially the spectrum of the excitational function and the tension of the tissues that is caused by external forces as well as by their own contraction. Additionally, the spectrum is influenced by the surrounding media, primarily the blood contained in heart and vessels. Last but not least, the intracardial blood volume itself represents a vibrational system, whose contribution to the heart sounds appears particularly distinct in the case of eddy flows caused by cardiac malfunctions, such as valvular stenosis or insufficiency. Due to the complexity of the heart it is not possible to formulate an analytical description of the vibrations or their spectra. Physical considerations and experimental studies, however, result in judgements of physiological or pathologic shifts of the PCG spectrum away from the normal distribution which are diagnostically relevant.

The well-known Laplace model describes the ventricles as a thin-walled hollow sphere. The tension $s$ of the wall depends on the trans-mural filling pressure $p$, the radius $r$ of the sphere, and the thickness $h$ of the wall, that is the myocardium:

$s=\frac{p \cdot r}{2 h}$

For thick-walled structures the relationship is:

$s(r)=\frac{p_{\mathrm{i}} r_{\mathrm{i}}^{3}\left(1+r_{\mathrm{a}}^{3} / 2 r^{3}\right)}{\left(r_{\mathrm{a}}^{3}-r_{1}^{3}\right)}$

where $r_{\mathrm{i}}$ and $r_{\mathrm{a}}$ represent inner and outer radii of the wall, and $r$ lies between these limits. The tension decreases from the inside towards the outside.

In the course of the heart cycle both wall tension and intracardial pressure and also the radius of the ventricles are subject to change. According to the stage of 
heart action, these three variables exchange the function of dependent and independent parameters. During diastole there is a passive increase of wall tension, the preload, caused by the filling pressure, whereas during the systole the contraction of the myocardium actively increases the wall tension (the afterload) and thus produces an interventricular rise of pressure. During the ejection time the tension of the myocardial wall is influenced essentially by the decrease in ventricular radius and the increase in its thickness. In view of the fact that the oscillation frequency of the myocardial wall depends on its tension, it becomes evident that pathologic changes of preload, afterload, and contractile force of the myocardial fibres (as well as dilatations) should result in characteristic shifts of the PCG spectrum, and thus are accessible to non-invasive diagnostics. Similar considerations are valid for pathologic conditions of the valves, which can be compared to vibrating membranes, where tension and size determine the oscillation frequencies. Besides pathologic variations of the PCG power spectrum, characteristic shifts are also to be expected according to physiological changes of the characteristic parameters that occur depending on gestational age and fetal maturity.

\section{Fetal maturity}

As during pregnancy all the frequency-determining parameters undergo physiological change - the growing dimensions and increasing contractile strength of the myocardium - characteristic changes of the PCG spectrum are to be expected as a function of the stage of fetal maturity. In order to verify this assumption, extensive experimental studies have been done. In order to compare different spectra using only one single parameter, the power spectra were integrated. The determining measure was the frequency within which $80 \%$ of the total PCG power is contained, i.e., where the power spectrum integral reaches $80 \%$ of its maximum. Mean values of the $80 \%$ mark for the first and second heart sound are shown in Fig. 2. Each point was computed from 30-50 fetuses of the same gestational age. Of course, mean values have been computed only from spectra of fetuses that were known to be

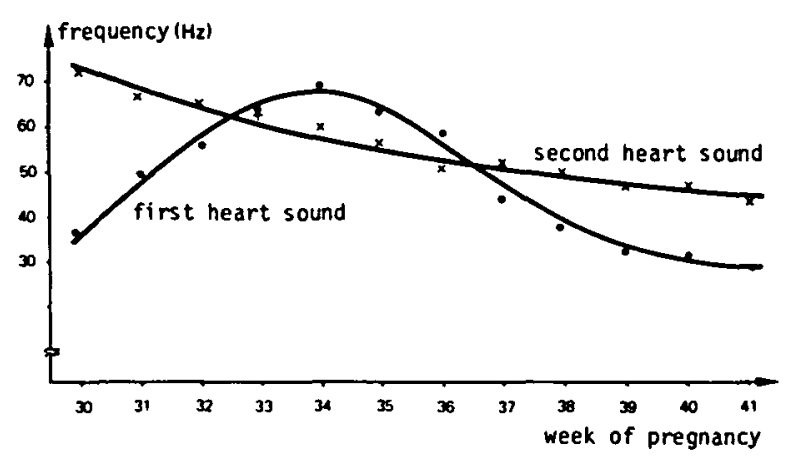

Fig. 2. Relationship between fetal PCG spectrum and gestational age. The traces show the mean $80 \%$ power marks of the integrated power spectra for the first and second heart sound dependent on the week of pregnancy. The standard deviation of the single measuring points typically corresponds to 1.4 weeks. 
developing normally. The first heart sound reveals a steady shift towards higher frequencies up to about the 34th week of gestation. Thereafter the shift reverses its direction. The spectrum of the second heart sound steadily shifts to lower frequencies. From physical models, the decrease in frequencies can be explained by the growth of the heart, whereas the ascending slope of the first heart sound's spectrum is primarily caused by the predominance of the increasing contractile strength of the myocardium during that stage of pregnancy.

On the basis of these findings, spectral analysis of the PCG can be considered a real alternative to better known measurements such as ultrasonic imaging in the determination of fetal maturity. Besides the information on physical dimensions, it also contains additional data on the function and the strength of the heart muscle. When investigating the reliability of this measurement, possible disturbing influences such as changing cardiovascular conditions (e.g. the heart rate) have to be considered. So far, however, no negative effects have been found. Indeed, measurements were always done at the basal heart rate with no labour activity, and in the absence of accelerations and decelerations. In several cases the spectra did not fit any stage of maturity, showing distinct deviations from normal values, and, without exception, further investigations (done partially after birth) confirmed the existence of heart diseases, predominantly valve malfunctions. This diagnostic capability is the direct consequence of the fact that each pathological variation of either cardiac function or anatomy results in a change in the PCG spectrum.

\section{PCG segmentation and momentary frequency analysis}

Conventional spectral analysis converts an interval of the phonocardiogram from the time domain to the frequency domain by Fourier transformation. Normally the interval corresponds to the time duration of the first or the second heart sound, but sometimes it is of the whole cardiac cycle. In this representation, however, the spectrum is a summary of all vibrations arising at any time within the interval, and detailed information on the different stages of contraction (e.g. the development or onset of single cardiac vibrations) is difficult to obtain. For exact diagnosis a continuous time-dependent measure of the vibrational mode of the heart is necessary. A convenient tool for this purpose is the momentary frequency of the PCG that is determined either by the Hilbert transform [4], or by the Wigner distribution function [5].

Analysis of the momentary frequency of the PCG yields much important information. The first heart sound marks the beginning of the systole. It consists of three segments, indicating the three different operational phases of the ventricles. The first segment results from the remodelling of the ventricles characterized by changing geometry and modest pressure rise. The main segment marks the steep increase of ventricular pressure occurring until the pulmonal and aortic valves are opening, signifying the onset of the ejection period and the third segment of the first heart sound. What is important in this context is the fact that there is no time interval where those parameters that determine the vibrational frequency - dimensions, volumes, and mechanical stress - are constant. As a consequence, we should not expect any particular interval of the heart sounds to be characterized by a 
constant power spectrum. Therefore any averaging spectral analysis of the PCG, such as Fourier transformation, is inadequate to separate the different functional stages, or to diagnose pathological variations of single segments. On the other hand, analysis of the momentary frequency provides information on the performance of each phase of the cardiac cycle and each part involved, namely the myocardium, the valves, the intracardiac blood volume and the vascular system. Moreover, the momentary frequency can indicate precisely the onset of the single operational phases of heart action, and is therefore best suited for the segmentation of heart sounds and the determination of fetal heart rate, as well as cardiac time intervals. There is actually no diagnostically relevant physiological parameter of the heart's mechanical action that cannot be judged from the phonocardiogram.

Distinct variations in the heart's vibration frequency are to be expected when the valves are opening or closing. The sudden changes of pressure and blood flow find a clear expression in the momentary frequency of the PCG. Whereas the time domain analysis hardly shows the opening and closing of the valves (e.g. the separation of aortic and pulmonal closure, which can only be seen if there is an increased delay of pulmonal closing, leading to a splitting of the second heart sound), momentary frequency-domain representation gives evidence of this event even under normal conditions. Fig. 3 shows as another example a first heart sound both in time- and

Original Signal $x(t)$

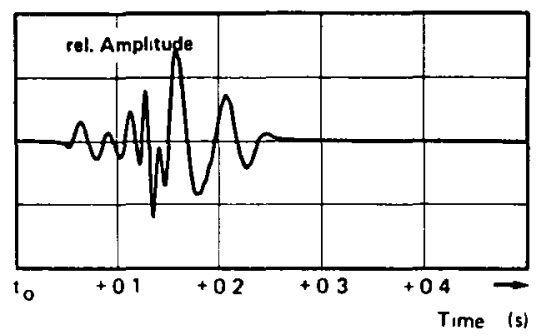

Orthogonal Signal $y(t)$

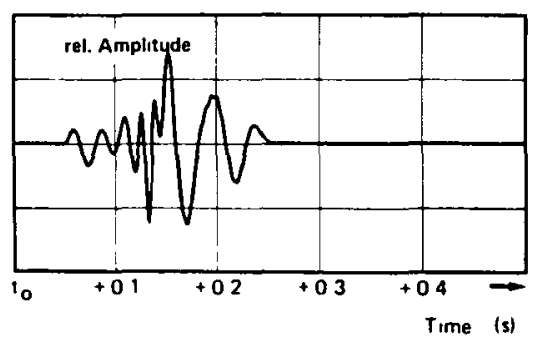

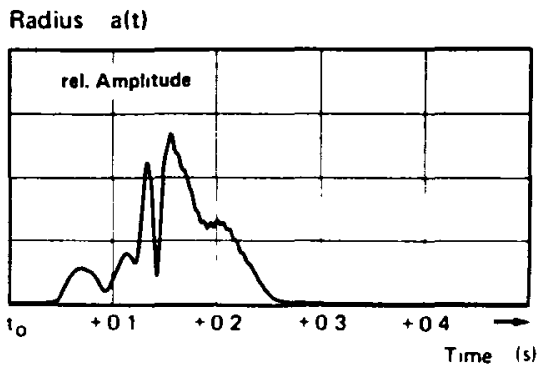

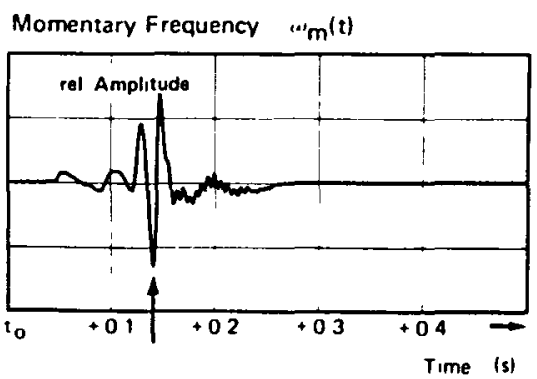

Fig. 3. Detection of aortic opening by momentary frequency analysis of the first heart sound In the original PCG, the opening of the valve cannot be detected unequivocally if the time is not known in advance. Orthogonal signal. radius. and momentary frequency are determined by the Hilbert transform. From the distinct negative maximum of the momentary frequency the aortic opening can be clearly detected 
momentary frequency-domain representation. The aortic opening can be detected unequivocally only by frequency analysis.

\section{Maternal cardiovascular condition and fetal movements}

With the onset of ejection time, a triple pulse wave travels along the arterial system; this consists of a pressure pulse, a flow pulse, and a diameter or volume pulse. A secondary effect of the pulse wave, which is rarely considered, is the generation of acoustic waves along the arteries. The pulse sound spreads within the body and, by recording it at the skin surface, the blood flow in the inner arteries can be monitored non-invasively. Due to the low-pass transfer function of blood vessels,
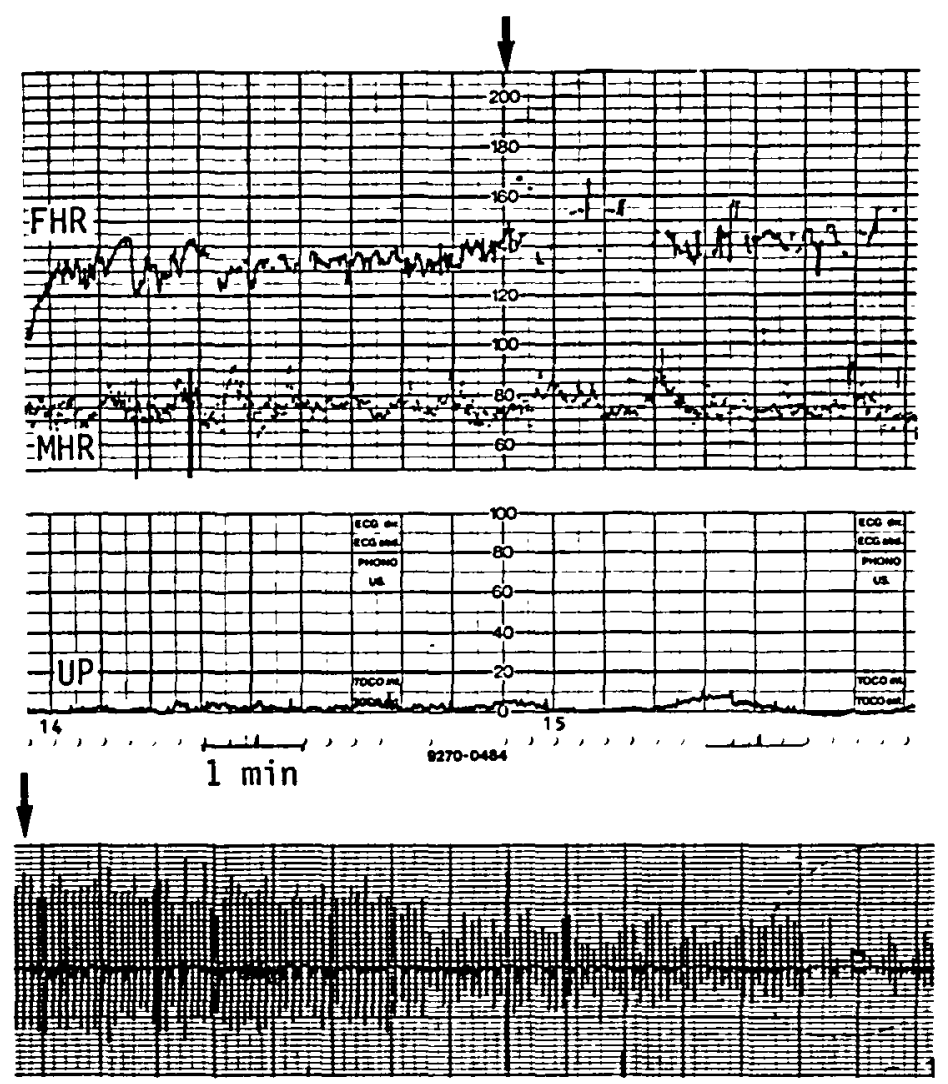

\section{maternal blood pressure}

Fig. 4. Fetal and maternal heart rate tracings together with an interval of maternal blood pressure recording, which starts at the time marked by an arrow in the CTG. The maternal blood circulation shows a disruption, resulting in a severe decrease of placental blood supply. FHR, MHR and maternal pulse were determined from the same abdominal acoustic signal. 
the sound of the blood flow becomes a lower-frequency signal with increasing distance from the heart. Thus only wide-band fetal phonocardiography will actually detect the abdominal maternal blood flow as an interfering signal component. Separation of this component from the PCG recording makes it possible to determine both maternal heart rate and, very approximately, the placental blood supply. Fig. 4 shows a simultaneous recording of a CTG together with maternal heart rate and abdominal maternal pulse. Both heart rates and the pulse have been determined from the phono signal. It can be seen that during the second half of the record the maternal heart rate remains fairly stable, whereas the placental blood supply is disrupted, resulting in severe fetal arrhythmia. Surveillance of the maternal circulation has been especially helpful in this case, as the FHR monitor did not indicate the risk situation. During the arrhythmia there was no valid FHR recording. A later, careful off-line analysis of the fetal PCG and ECG, both stored on magnetic tape, clearly showed the fetal arrhythmia, which was obscured in the FHR tracing due to the unsatisfactory quality of heart rate determination during this arrhythmia.

According to Talbert et al. [1], analysis of the lowest-frequency band of the abdominal acoustic signal $(0.3-1.5 \mathrm{~Hz})$ is able to show fetal trunk and limb movements as well as breathing movements, where the signal can even identify the inspiratory and expiratory waveforms of each breath.

\section{Conclusion}

A new generation of transducers for the recarding of the fetal phonocardiogram, characterized by extremely high sensitivity and high signal-to-noise ratio, extended bandwidth, and reduced liability ta interference, provides a completely new approach to fetal monitoring. New methods of time- and frequency-domain processing of the acoustic signal increase the diagnostic potential of phonocardiography over and above the previous state of knowledge. Not only the fetal heart rate, but also very detailed information on fetal and maternal cardiovascular condition, on heart and valve diseases, and on fetal maturity and breathing is made available for prenatal diagnostics.

\section{References}

1 Talbert DG, Davies WL, Johnson F, Abraham N. Colley M, Southall DP Wide handwidth fetal phonography using a sensor matched to the compliance of the mother's abdominal wall. IEEE Trans Biomed Eng 1986: 33 : 175-181.

2 Nagel JH. The spectrum of the fetal PCG as an indicator of fetal maturity. In Rolfe P. ed. Fetal and Neonatal Physiological Measurements. London, Butterwarth \& $\mathrm{Co} ; 1986$ : in the press.

$3 \mathrm{Nagel} \mathrm{JH.} \mathrm{Progress} \mathrm{in} \mathrm{fetal} \mathrm{monitoring} \mathrm{by} \mathrm{improved} \mathrm{data} \mathrm{acquisition} \mathrm{and} \mathrm{interpretation.} \mathrm{IEEE} \mathrm{EMB}$ Mag 1984; 3, No. 3: 9-13, 1984.

4 Bodecker RH. Anwendbarkeit frequenzanalytischer Methoden in der Phonokardiographie Gießen, Verlag der Ferber'schen Universitätsbuchhandlung: 1982.

5 Bartelt HO, Brenner K-H, Lohmann AW. The Wigner distribution function and its optical production Optics Commun 1980; 32: 32-38. 